\title{
Strategic asset allocation using quadratic programming with case based reasoning and intelligent agents
}

\author{
E. Falconer, A. Usoro, M. Stansfield \& B. Lees \\ School of Computing, University of Paisley, Scotland, UK
}

\begin{abstract}
There is an abundance of intelligent agent decision support systems (DSS) that have been used to assist with portfolio management decisions. These systems have tended to focus on fundamental, technical and trader behaviour analyses whereas efficient markets hypothesis, as argued by researchers such as Clarke et al., suggest that effort should rather be spent on strategic asset allocation. All of the artificial intelligent studies that have been found have focused on analysis methods which are aimed at identifying investment opportunities with above average returns. According to Clarke et al, although giving support to these types of decision is possible with today's computing power, to be effective these systems must identify an opportunity before the rest of the marketplace. There is evidence from the efficient markets hypothesis that the stockmarket reflects new information so quickly that these types of analysis technique are ineffective. As an alternative to these methods Markowitz developed an approach known as mean-variance efficiency analysis which aimed at allowing an investor to gain a specific level of return for a corresponding degree of risk. Sharpe introduced an alternative way of implementing meanvariance efficiency theory by defining stocks and other investments in terms of asset classes. A literature search has revealed that there has as yet been no attempt to investigate this potential. This paper therefore aims to fill this gap by reporting on research that involves the incorporation of quadratic calculations in an intelligent agent and case based reasoning tool for strategic asset allocation. This paper provides the results of an evaluation into the effectiveness of intelligent agents and case based reasoning in dealing with this problem.

Keywords: intelligent agents, quadratic optimization, portfolio management, asset allocation, case based reasoning.
\end{abstract}




\section{Introduction}

The efficient markets hypothesis has led academics and practitioners to divide investment management approaches into two primary types: i) active investment management, where an investment manager uses available information and forecasting techniques to seek better performance than a diversified portfolio; and ii) passive investment management, where an investment manager has minimal expectations about the returns that can be achieved and relies instead on diversification to match the performance of a market index [1,2]. Although each of these approaches has benefits, there has been strong evidence to support the claim that the profitability of active investing is minimal, especially after transaction costs, and therefore passive investing is more profitable $[1,3,4]$. One of the implications of choosing a passive investment approach is that portfolio managers must concentrate on other strategies for improving the return from their portfolio [1]. Examples of these strategies include cost management, diversification and asset allocation strategies. This research concentrates on asset allocation.

There exist many different approaches to asset allocation. These variations include strategic, tactical and dynamic asset allocation, amongst others. This research concentrates on strategic asset allocation. The optimal strategic asset allocation can be determined in one of two ways: by maximizing return for a given risk level or alternatively, minimizing risk for a particular return objective [5-7]. A strategic asset allocation decision is made up of a quadratic component and a utility component [5]. The quadratic component is concerned with constructing the portfolio under quadratic constraints whilst the utility component is concerned with determining the investor's attitude towards risk. An earlier stage of the work described in this paper was presented at the International conference on intelligent agents, web technologies and internet commerce - IAWTIC - in Vienna Austria [8].

This research considers the application of a combined intelligent agent and case based reasoning (CBR) approach to the strategic asset allocation problem. Broadly speaking, literature on intelligent agent and case based reasoning in finance has focused on three areas: i) portfolio monitoring, ii) stock selection, and iii) behavioural finance. Portfolio monitoring is defined as the ongoing, continuous, daily provision of an up-to-date financial picture of an existing portfolio [9]. Stock selection is defined as an analysis technique which uses valuation and forecasting techniques to identify mis-priced assets [10]. Behavioural finance is defined as the computational study of economies modelled as evolving systems of autonomous interacting agents. The determination of an investor's utility or support of quadratic programming does not appear to have been considered. This research therefore assesses the effectiveness of intelligent agents and CBR in dealing with the complexities associated with this problem. This paper concentrates on the quadratic program calculations under mean variance efficiency. Utility theory will be considered in a subsequent paper. 
The paper reports preliminary results on the effectiveness of a prototype CBR and agent-based system towards the quadratic programming problem. The rest of this paper provides: (i) a background to the research problem and an overview of the data preparation steps; (ii) an overview of agent and CBR characteristics; (iii) an outline of the agent architecture; (iv) an evaluation of case based reasoning as it applies to the problem and preliminary results on the intelligent agent approach; (v) conclusions and future directions.

\section{Agent and CBR characteristics}

Strategic asset allocation is characterised by a variety of complexities that have to be considered in the design of a decision support system [5]. These complexities are related to the problem of matching investors with portfolios. Some of these complexities are listed below.

(i) The strategic asset allocation task is distributed over various parts; this means that different factors contribute to the matching of an investor with a portfolio.

(ii) Some tasks require input from other tasks to arrive at a conclusion; thereby necessitating the need to share data and control the flow of information between tasks.

(iii) There is a chance that the same set of data inputs could be used multiple times; this means there is a potential for task duplication.

(iv) There is not one solution that can be applied to every set of circumstances; this means that each portfolio or investor could be either unique or at best similar but often not exactly the same as those encountered previously.

(v) In order to match up an investor with a portfolio it is necessary to have input from a user; and this user could require a different level of assistance depending on their preferences.

In the light of these observations a review was conducted of available technologies and intelligent agent and case based reasoning identified as displaying the types of attribute that could deal with the complicated nature of the strategic asset allocation decision. Agent and CRB technologies have been used to successfully deal with the complexities of portfolio management $[9,11,12]$.

\subsection{Intelligent agents}

Intelligent agents are software artefacts that have a number of characteristics that distinguish them from other technologies. In particular, an agent can be given a goal or task which it can complete autonomously [13]. Agents may also carry out tasks or goals in parallel with the existing computer operations and ask for advice from humans if they become "stuck" in a problem [14]. Agents can act proactively and communicate with human users, providing a personalised service to some users and communicating with other agents in a multi-agent environment [13]. Wooldridge [14] discusses how intelligent agents can interact with, and react to, a user's preferences. This means that software can be 
designed to recognise a user's actions and modify the user/agent interaction to ensure the level of support is appropriate to that user.

Decker and Sycara [9] also discuss how intelligent agents can incorporate a separate control component to regulate the flow of information between distributed tasks. This control component can organise the flow of information that passes between the various parts of the problem and ensures that a conclusion is reached. This ability to control distributed tasks is a key attribute of the anticipated solution. Intelligent agents therefore demonstrate various abilities that suggest they may be appropriate for resolving this problem. In particular the ability of intelligent agents to interact with human experts, organise data flow, construct knowledge bases and learn from experience are perceived to be important for overcoming the complexities associated with matching portfolios with investors.

\subsection{Case based reasoning}

Case-based reasoning (CBR) is a methodology that can allow software to learn from experience. Case based reasoning subdivides a problem into a series of tasks and then combines the results of each task to form a case. Each case is then compared with previous cases to determine whether new experiences can be learned. Althoff [15] identifies four tasks that exist as a part of most general CBR programs: retrieve task - to retrieve similar cases; reuse task - to reuse information and knowledge from previous cases to solve a problem; revise task where the problem is revised in line with previous experiences; retain task where experience is retained that could be used for future cases. Althoff [15] also discusses how CBR systems gather case data from distributed tasks and join the outputs of these tasks together to formulate a combined state. In this way a distributed problem can be resolved, with each distributed solution contributing to all future solutions.

Althoff [15] also discusses how CBR systems can be used to subdivide each solution into different parts and then pass requests for analysis onto other components. By initiating this task division CBR software can perform distributed tasks at the same time as other tasks are performed and then combine the parts to arrive at a more complete solution. Althoff [15] identifies that CBR systems evaluate each set of results to determine whether the current case is new, or whether it matches a previous case. CBR was found to offer additional capabilities that would strengthen the intelligent agent approach. It is the ability of CBR to organise distributed tasks, subdivide solutions into categories, historically evaluate solutions and learn from experience which means it may be applicable for dealing with the complexities associated with strategic asset allocation. 


\section{Preliminary results}

There are two sets of results to report, findings related to: (i) the quadratic component and the use of agents to overcome the limitations of infeasible solutions; (ii) the application of CBR to the quadratic programming problem.

\subsection{The quadratic component and the quadratic program}

In contrast to the standard approach the securities in this case are in the form of global asset prices. The global approach differs slightly from the standard asset allocation approach as the focus is upon prices that reflect a certain global market. In contrast, asset allocation approaches usually focus on much broader asset allocation classes, such as real estate, stocks, bonds and cash. Despite the differences, there are many advantages to adopting a global approach. This decision is supported by the globalisation of markets discussed in literature and the importance of global diversification [16-20]. The historical global asset prices were obtained from Morgan Stanley Corporate International (MSCIC). The calculation used global equity asset classes for 9 specific asset classes. However, although the assets are limited, the model could easily be extended to include additional asset classes, cash assets or bond assets, with minimal effort. The QP used a quadratic function to determine the optimum investment amount to place in each asset class. The following objective function was used to maximise return or minimise risk (from an article by Fylstra [21].

$$
\begin{array}{ll}
\text { Maxmise } & c^{T} x-x^{T} Q^{o} x \\
\text { Subject to: } & A x>=b \\
& \Sigma x=1 \\
& x i>=0 \text { for all } i .
\end{array}
$$

$Q^{o}$ is a matrix containing covariances for the assets held within the portfolio. $c^{T}$ is a vector containing returns on each of the assets held within the portfolio. $A$ is also a vector containing the returns on each of the assets held within the portfolio. $b$ is a value that represents the minimum portfolio return. $x$ is the amount to be invested in the $i$ th asset class within the portfolio. The data tables are available from the author by request. A front end application was developed and is illustrated in Figure 1. The application allows a user to choose asset classes, choose a minimum portfolio return, view covariances and mean returns, and view the optimised result. Yet despite the initial simplicity of the quadratic program in calculating the optimum results, from close inspection, it was apparent that at times it was not possible to produce results that were useful. An extensive evaluation was therefore conducted of the quadratic program and the outputs that were produced under various constraints. This problem is discussed by Fylstra [21] who indicates that analysts take great care to ensure that their portfolio models remain calculable under quadratic or linear constraints. The following four categories identify the types of result that were returned. 


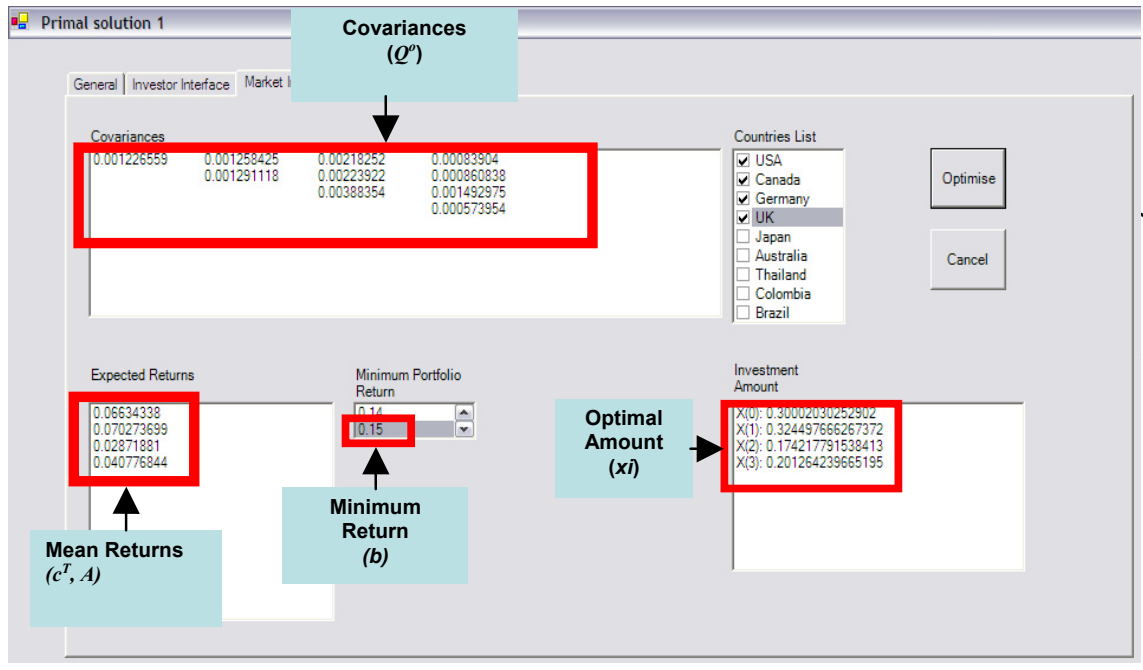

Figure 1: Front end application for portfolio choice.

It was found from the evaluation that each quadratic result fell into one of these four categories. However, if category two, three or four occurred the user was required to change their choice. In order to resolve this problem intelligent agents are being used to search for alternative solutions. Multiple agents are incorporated in the solution to conduct parallel searches for more effective solutions. The aim of each intelligent agent is to determine alternatives which contain either different asset classes or a different minimum portfolio return.

The closeness of the solution is considered in terms of the distance between the optimum investment amounts returned by each of the amounts invested. The intelligent agent systems attempt to define alternative solutions by modifying the minimum portfolio return or adjusting the asset classes within the solution, reinitiating the quadratic calculations through the MOSEK tool and therefore resolving the problem.

The agent tasked with adjusting the minimum portfolio return keeps the asset classes the same and simply amends the portfolio return to achieve feasible results. On the other hand the agent system focuses on adjusting the asset classes held within the portfolio by using four factors to determine the similarity of each country with another: global influence, average volume, length established and emerging rating. These factors are used to determine the suitability of each country as an alternative to other countries chosen by the user. A numeric value was determined for each country factor which allowed a comparison of asset classes to occur.

The agent adjusting the minimum portfolio return and the asset classes used threshold values to determine the suitability of alternative choices. From an evaluation of the quadratic results it was determined that a threshold value of 0.02 was appropriate for the minimum portfolio return. In so doing it was possible in most cases to determine an alternative portfolio. The agent changing 
the chosen asset classes also incorporated a threshold that was set for each of the similarity factors (mentioned above as global influence, length established, average volume and emerging rating). Using the similarity factor and the threshold value the agent program attempts to determine an asset class or asset classes that are sufficiently similar. The agent program proceeds to optimise the result and determine whether the portfolio is more suitable.

Results of an evaluation of the agent program in achieving these aims shows that in most cases an alternative solution can be found. By incorporating multiple agents into the solution it is therefore possible to overcome the limitations of the quadratic program.

\subsection{Case based reasoning search and retrieve method}

This section outlines the development of the case-based-reasoning solution and the identification of the case features. The CBR program operates by first having the user provide the system with a set of responses in relation to risk and the user is asked to choose a portfolio for this type of investor. The system uses the quadratic program to provide the problem and solution variables to the CBR component. The CBR program then evaluates and monitors cases as new problems and solutions are introduced. In general the CBR tool carries out the following steps:

1. Searches from its memory to find portfolios that have been previously chosen for certain investors and makes an assessment of similarity.

2. Infers an answer from the most similar matches.

3. Adjusts the portfolio to investor matching solution for changes in circumstances by inferring relationships between sets of historical data.

4. Modifies solutions from step three and records them for future use.

The search and retrieve stages are completed in the first and second steps and will use the nearest neighbour retrieval method. The nearest neighbour retrieval method identifies possible matches by defining values that can be used to index each case. The indexes are then weighted in terms of importance. Using the nearest neighbour retrieval method there are three states that the CBR program can find itself in when evaluating alternatives: (i) an exact match where the same problem has been used before; (ii) no match where the current problem does not match any previous case; or (iii) a partial match where the current case is similar but not exactly the same as a previous case. The features that relate to this particular problem have been identified and are shown in Table 1. Each feature has been allocated a weight based on the importance of that feature to the problem. Therefore for instance the country name and number of countries (given a score of 10) are considered more important than global influence, average volume (given a score of 5). Each feature either relates to an aspect about either the portfolio or the investor.

The portfolios will be given a score for each portfolio and investor combination. This score is then used to determine how close this case may be to previous cases. The values will be interpreted by considering the potential risks associated with the individual portfolio as it compares with other portfolios. 
Table 1: $\quad$ Case Features as they apply to the quadratic problem and investor profiler.

\begin{tabular}{|l|c|c|c|}
\hline Rule Description & Exact Match & Partial Match & No Match \\
\hline CountryName & 10 & 0 & 0 \\
\hline Number of Countries & 10 & 5 & 0 \\
\hline Global Influence & 5 & 2 & 0 \\
\hline Average Volume & 5 & 2 & 0 \\
\hline Length Established & 5 & 2 & 0 \\
\hline Emerging Rating & 5 & 2 & 0 \\
\hline Covariances & 10 & 5 & 0 \\
\hline Minimum Portfolio Return & 10 & 5 & 0 \\
\hline Mean Return & 10 & 5 & 0 \\
\hline Mosek Result & 10 & 5 & 0 \\
\hline Risk Attitude & 10 & 5 & 0 \\
\hline Risk Perception & 10 & 5 & 0 \\
\hline Knowledge and Experience & 5 & 2 & \\
\hline Optimal Risk Level & 5 & 2 & 0 \\
\hline Complexity & 10 & 7 & 0 \\
\hline Investment Goal & 10 & 5 & 0 \\
\hline Investment Objective & 10 & 5 & 0 \\
\hline Investment Time Horizon & 10 & 7 & 0 \\
\hline Investment Amount & 10 & 7 & 0 \\
\hline Estimated Year Return & 5 & 2 & 0 \\
\hline Investor Expected Return & 5 & 2 & 0 \\
\hline Total & 180 & & 0 \\
\hline
\end{tabular}

The following is an example of the CBR process. During the first step the user is presented with a set of responses and is asked to choose an asset class and a minimum portfolio return. In this example the user chooses the following portfolio for their investor: Asset classes: USA, UK, Germany and Canada; minimum portfolio return: 0.15 . During the second stage all previous cases are compared with the portfolio chosen in step 1 for the type of investor identified by the user. This retrieval is based on the values identified in table 1. The CBR program initially finds three portfolios that are similar to the current investor. The first portfolio contains USA, UK, Thailand and Canada and has the same portfolio return as the current portfolio. The investor has some similarities to the current investor however the time horizon and risk profile are different. This portfolio/investor combination therefore achieves a $58 \%$ similarity based on the scores received. The second portfolio contains USA, UK, Canada and has a different portfolio return. However, the investor has different goals and investment amount. The second portfolio/investor combination achieves a $60 \%$ similarity. The third portfolio contains USA, UK, Canada, Germany and has a different portfolio return. The investor however is very similar to the current set of investor responses provided by the investor. The third portfolio/investor combination achieved an $87 \%$ similarity. The final stage is based on the results of the previous step where the alternatives are evaluated. In order for the current problem to be identified as a possible match the minimum-portfolio-return and the features relating to the investor are all required to be very close to that of the current case. All three portfolios are displayed as possible matches to the user with the score displayed for each portfolio. The final stages of general CBR cycles involves revising the proposed solution and updating the case base and retaining experience. As yet these have not been considered and will be reported at a later stage. 


\section{Conclusions and future direction}

This paper outlines a decision-support system to assist strategic asset allocation decisions based on an intelligent agent. The system uses case-based reasoning to make portfolio optimisation calculations under quadratic constraints. These calculations are performed as part of an optimisation of the expected return and the minimisation of risk within a portfolio of broad asset classes.

The results attained from an evaluation of the quadratic program present sufficient evidence to support the decision to use intelligent agents for the problem of matching investors to portfolios. In addition to this the ease with which the variables associated with this problem fit with the general approach to CBR also support the decision to use CBR within the approach.

Combining the capabilities of intelligent agent and CBR based systems therefore present the opportunity to support strategic asset allocation decisions. By combining these technologies the system can learn from its interactions with the user and also its experiences when evaluating problems.

The next piece of work to be undertaken will involve extending out the CBR component and carrying out further validation on the agent program. Additional work is also being undertaken to identify more opportunities for using the CBR and agent programs to provide more assistance.

\section{References}

[1] Clarke, J; Mendelker, G; Jandik, T (2001) Expert Financial Planning : Investment Strategies from Industry Leaders, (Edited by Robert C Arffa), 2001, chapter 10, Wiley and Sons.

[2] Fama E.F (1991) "Efficient Capital Markets: II", Journal of Finance, December.

[3] Bernstein, W (2000) The Intelligent Asset Allocator: How to Build Your Portfolio to Maximize Returns and Minimize Risk ; McGraw-Hill Education.

[4] Bernstein, W (2002) The Four Pillars of Investing: Lessons for Building a Winning Portfolio, McGraw-Hill Education.

[5] Nemati, H; Iyer, L.S (1999) "Intelligent decision support system for asset allocation", Americas Conference on information Systems.

[6] Kaplan, P (1998) "Asset Allocation Models Using the Markowitz Approach", http://www.misp.it/doc/materiali_doc/Curti1-EN.pdf, accessed $17^{\text {th }}$ February 2006.

[7] Todd, G.P; Markowitz, H (2000) Mean-Variance Analysis in Portfolio Choice and Capital Markets, Frank J. Fabozzi Associates.

[8] Falconer, E; Usoro, A; Stansfield, M; Lees, B (2006); A Prototype Intelligent Agent Based DSS for Quadratic Portfolio Optimization Calculations with Case Based Reasoning, Agent, Internet and Web Technologies International Conference, Vienna Austria. 
[9] Decker, K.S; Sycara, K. (1997) "Intelligent Adaptive Information Agents", Journal of intelligent Information Systems, Kluwer Academic Publishers, volume 9, pp $239-260$.

[10] Luo, Y; Davis, D; Liu, K (2002) “A multi-agent system framework for decision making support in stock trading", The IEEE Network Magazine Special Issue on Enterprise Networking Services, Volume 16, No 1, $\mathrm{Jan} / \mathrm{Feb}$.

[11] Davis, D; Luo, Y (2001) "Using KADS to Design a Multi-Agent Framework for Stock Trading", Multi-Conference Event - Agent for EBusiness on the Internet, Las Vegas, June 2001.

[12] Davis, D; Luo, Y; Liu, K (2000) “A Multi-Agent Framework for Stock Trading”, World Computing Conference, Beijing, August.

[13] Turban, E; Aronson, AE (2001) Decision Support Systems and intelligent systems, Sixth Edition, Prentice-Hall Incorporated.

[14] Wooldridge. M (2002) An Introduction to Multiagent Systems, John Wiley and Sons Ltd.

[15] Althoff, K (2001), "Case-Based Reasoning”, ICCBR-01 Workshop On Case-Based Reasoning Authoring Support Tools, 31st July.

[16] French, K (1991) "Investor Diversification and International Equity Markets", American Economic Review, MIT Press.

[17] Lummer, S; Riepe, MW (1994a) "Taming Your Optimisation: A Guide through the pitfalls of Mean Variance Optimisation", Global Asset: Techniques for Optimising Portfolio Management, Edited by J.S. Lummer, M.W. Riepe, , John Wiley \& Sons.

[18] Lummer, S; Riepe, MW (1994b) "The Role of Asset Allocation in Portfolio Management", Global Asset Allocation: Techniques for Optimising Portfolio Management, Edited by J.S. Lummer, M.W. Riepe, John Wiley \& Sons.

[19] Kaplan, P (1998) "Asset Allocation Models Using the Markowitz Approach”, http://www.misp.it/doc/materiali_doc/Curtil-EN.pdf, accessed $17^{\text {th }}$ February 2006.

[20] Dempster, Germano, Villaverde, (2003) "Global Asset Liability Management”, British Actuarial Journal, Volume 9, Number 1, pp. 137-195(59)

[21] Fylstra, D (2005) "Introducing Convex and Conic Optimization for the Quantitative Finance Professional”, Wilmott Magazine, pp. 18-22. 